\title{
Noninvasive assessments of oxygen delivery from the microcirculation to skin in hypothermia-treated asphyxiated newborn infants
}

\author{
Siv Fredly ${ }^{1,2}$, Drude Fugelseth ${ }^{1,2}$, Cathrine S Nygaard' ${ }^{1}$ E. Göran Salerud ${ }^{3}$, Tom Stiris ${ }^{1,2}$ and Knut Kvernebo ${ }^{2,4}$
}

\begin{abstract}
BACKGROUND: Therapeutic hypothermia (TH) has become standard treatment for severe and moderate hypoxic-ischemic neonatal encephalopathy (HIE). Our group has developed an optically based, noninvasive concept of assessing the capacity for oxygen delivery from the microcirculation to the cells of a tissue under investigation. The hypothesis was that mechanisms of reduced oxygen delivery due to reduced metabolism in cooled asphyxiated neonates could be characterized with this concept.
\end{abstract}

METHODS: The skin of 28 asphyxiated newborn infants was studied on days 1 and 3 during TH and on day 4 following rewarming with laser Doppler perfusion measurements (LDPM), computer-assisted video microscopy (CAVM), and diffuse reflectance spectroscopy (DRS). Twenty-five healthy neonates served as a control group.

RESULTS: The LDPM decreased during cooling $(P<0.01)$. Functional capillary density was higher both during and following TH compared with control infants $(P<0.01)$. Capillary flow velocities were reduced during TH $(P<0.05)$. The heterogeneity of the flow velocities was larger in the HIE infants than in the control infants. Tissue oxygen extraction was higher during TH $(P<0.01)$.

CONCLUSION: This study indicates that assessments of skin microvascular density, capillary flow velocity, and oxygen extraction can be used to characterize reduced oxygen delivery to cells during $\mathrm{TH}$.

$\mathbf{T}$ herapeutic hypothermia $(\mathrm{TH})$ has become the standard treatment for severe and moderate hypoxic-ischemic neonatal encephalopathy (HIE), as a means of reducing mortality and cerebral morbidity (1-4). TH decreases the metabolic rate by $6-10 \%$ per degree celsius $\left({ }^{\circ} \mathrm{C}\right)$ drop in core temperature $(5,6)$.

In critically ill infants, hemodynamic disturbances and shock may develop. One definition of shock is the inability of the circulatory system to deliver sufficient oxygen to sustain cellular metabolism and organ function (7). Although the oxygen delivery takes place at the capillary level, few studies regarding microvascular function in infants have been published, most likely due to the complexity of the microvasculature and the lack of appropriate technology for the noninvasive bedside examinations of easy accessible organs such as the skin, eyes, and oral cavity.

In adult skin, the microvessels are arranged into a superficial papillary nutritive layer fulfilling the metabolic demand of epithelial proliferation and a deep and a superficial horizontal plexus serving body thermoregulation. In newborns, the vascular architecture is not fully differentiated and consists of a disordered network with horizontal microvessels (8). Laser Doppler perfusion measurement (LDPM) allows for the quantification of blood cell flux in tissue volumes of approximately $1 \mathrm{~mm}^{3}$. This technique has been commercially available for more than $30 \mathrm{y}$ but is rarely used routinely in any clinical discipline due to its inability to differentiate between the skin's superficial nutritive- and deep thermoregulatory perfusion. LDPM may be more valuable in the skin of neonates because of the less differentiated vascular structures $(8,9)$.

Our group has developed an optically based, noninvasive concept for assessing the capacity of oxygen delivery from the microcirculation to cells of a tissue under investigation. Data are collected using computer-assisted video microscopy (CAVM) and diffuse reflectance spectroscopy (DRS). Analyzing recorded data files, parameters related to capillary density, capillary flow velocities, and microvascular oxygen saturation may be extracted. These parameters can be used to assess the capacity for oxygen delivery from the microcirculation to the cells in the tissue under investigation. In a previous paper, we demonstrated that this concept may be used to obtain reproducible data in healthy newborn infants (8).

The hypothesis for this study was that reduced oxygen delivery due to reduced metabolism in cooled asphyxiated neonates can be detected and characterized with a combination of LDPM, CAVM, and DRS. Furthermore, we wanted to describe the changes in microvascular morphology and function caused by cooling.

\footnotetext{
'Department of Neonatal Intensive Care, Oslo University Hospital, Ullevål, Oslo, Norway; ${ }^{2}$ Institute of Clinical Medicine, Faculty of Medicine, University of Oslo, Oslo, Norway; ${ }^{3}$ Department of Biomedical Engineering, Linköping University, Linköping, Sweden; ${ }^{4}$ Department of Cardiothoracic Surgery, Oslo University Hospital, Ullevål, Oslo, Norway. Correspondence: Siv Fredly (siv.fredly@online.no)

Received 8 July 2015; accepted 25 November 2015; advance online publication 2 March 2016. doi:10.1038/pr.2016.16
} 


\section{RESULTS}

Twenty-eight asphyxiated newborn infants were included in the present study (Table 1). The survival rate was high (93\%). One infant died within the first $24 \mathrm{~h}$ of life due to multiorgan failure, and another infant died at day 27 of life after redirection of care due to severe clinical and cerebral MRI pathology. Six infants exhibited severe MRI pathology, and 13 infants minor MRI pathology on day 11. Seven infants had completely normal MRI findings. During cooling, 10 infants developed either sepsis or symptoms of infection (35.7\%).

Heart rate decreased by $25 \%$ during $\mathrm{TH}$, the only central hemodynamic parameter with a significant change (Table 2). Serum lactate was initially high, but improved rapidly during TH. Following rewarming, oxygen saturation decreased from 97\% to approximately $94 \%$ (Table 2 ).

\section{Microcirculatory Assessments}

In all subjects, it was possible to obtain the microvascular data on day $1(\mathrm{~d} 1)$ and day $3(\mathrm{~d} 3)$ during cooling and on day $4(\mathrm{~d} 4)$ following rewarming. The examinations were performed over 30-45 min without interfering with ongoing therapy.

Table 1. Data of the asphyxiated study population

\begin{tabular}{lc}
\hline Values median (range) or number (percentage) & $\begin{array}{c}\text { Total population } \\
(n=28)\end{array}$ \\
\hline Gestational age, week & $39.7(36.0-41.9)$ \\
Birth weight, g & $3,486(2,117-5,200)$ \\
Inborn, $n$ (\%) & $15(53.6)$ \\
Cesarean section, $n$ (\%) & $11(39.3)$ \\
5-min Apgar Score & $3(0-7)$ \\
10-min Apgar Score & $5(0-9)$ \\
Sex, male, $n$ (\%) & $17(60.7)$ \\
pH in umbilical artery & $6.92(6.59-7.19)$ \\
Lactate in umbilical artery, mmol/l & $15.5(5.0-29.0)$ \\
Base deficit in umbilical artery, $\mathrm{mmol} / \mathrm{l}$ & $13.7(4.5-24.0)$ \\
Respiratory support $(n(\%))$ & $25(89.3)$ \\
Inotropes, $n(\%)$ & $16(57.1)$ \\
Sarnat 3 at start, $n(\%)$ & $22(78.6)$ \\
Clinical seizures, $n$ (\%) & $12(44.4)$ \\
\hline
\end{tabular}

Table 2. Data from the asphyxiated infants during (day 1 and 3 ) and after (day 4) hypothermia treatment

\begin{tabular}{lccc}
\hline Values (mean (SD) & Day 1 & Day 3 & Day 4 \\
\hline MAP, mmHg & $45.1(5.7)$ & $45.5(5.3)$ & $45.7(6.6)$ \\
Saturation, \% & $96.7(2.7)$ & $97.1(2.4)$ & $94.3(2.6)$ \\
Pulse, beats/min & $96.5(12.0)$ & $96.5(15.1)$ & $129.6(12.9)^{*}$ \\
Lactate, $\mathrm{mmol} / \mathrm{I}$ & $14.2(5.9)$ & $1.5(1.0)^{*}$ & $1.5(1.2)^{*}$ \\
Core temperature, ${ }^{\circ} \mathrm{C}$ & $33.5(0.1)$ & $33.5(0.1)$ & $36.6(0.5)$ \\
Skin temperature, ${ }^{\circ} \mathrm{C}$ & $31.9(0.4)$ & $31.9(0.5)$ & $34.9(0.6)$ \\
\hline MAP, mean arterial blood pressure. & & \\
${ }^{*} P<0.001$. & & &
\end{tabular}

LDPM. LDPM decreased significantly during cooling compared with rewarming (Figure 1). There was no difference in LDPM in asphyxiated newborns following rewarming compared to the healthy controls. The heterogeneity of LDPM perfusion among seven nearby repeated measurements, expressed as a coefficient of variation $(\mathrm{CoV})$, did not differ between controls and asphyxiated infants, or within the patient group from $\mathrm{d} 1$ to $\mathrm{d} 4$ (patients $\mathrm{d} 1: 18.8 \%$ (7.7-63.2), d3: 22.4\% (9.0-52.7), d4: $27.3 \%$ (6.0-45.7), and controls: $21.8 \%(6.6-54.1)$ ).

CAVM. Mean functional capillary density (FCD) was significantly higher both during cooling and after rewarming in the asphyxiated infants compared with the healthy controls (Figure 2). The heterogeneity of FCD among five repeated measurements, expressed as $\mathrm{CoV}$, did not change within or among groups (patients $\mathrm{d} 1: 12.0 \%$ (4.6-17.6), d3: 11.6\% (3.219.4), d4: 9.6\% (5.3-20.4), and controls: $13.0 \%$ (4.0-24)).

The control group had capillary flow velocities predominantly in category three and four (continuous low and high flow) (Figure 3). Rewarmed asphyxiated infants had similar findings with the exception of a $15 \%$ reduction in category

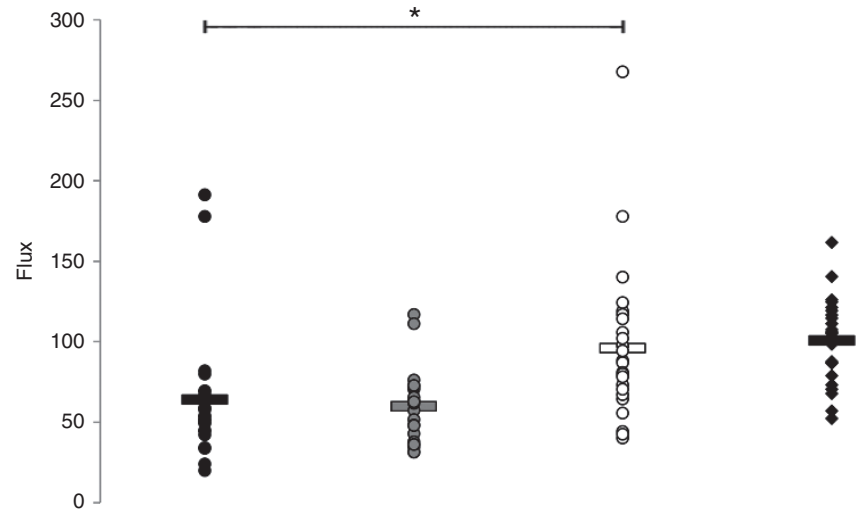

Figure 1. Laser Doppler perfusion. Asphyxiated newborn infants $(n=28)$; during hypothermia day $1=$ black circle; during hypothermia day 3 = gray circle; after rewarming day $4=$ white circle. Healthy normothermic controls $(n=25)=$ black route. ${ }^{*} P<0.01$.
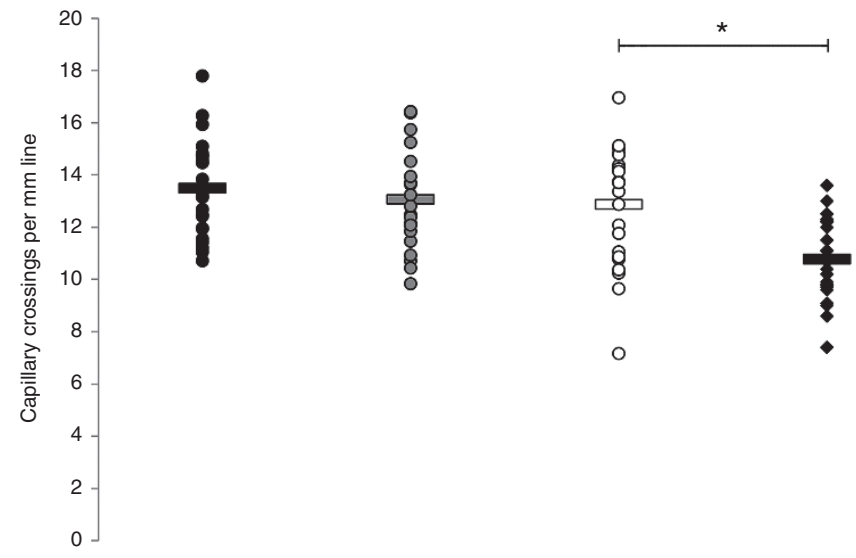

Figure 2. Functional capillary density. Asphyxiated newborn infants $(n=28)$; during hypothermia day $1=$ black circle; during hypothermia day 3 = gray circle; after rewarming day $4=$ white circle. Healthy normothermic controls $(n=25)=$ black route. ${ }^{*} P<0.01$. 


\section{Articles $\mid$ Fredlyetal.}

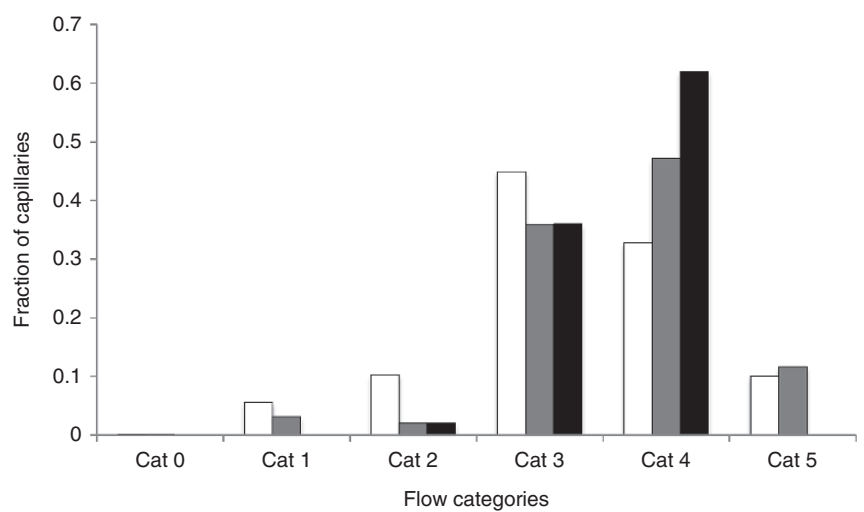

Figure 3. Fraction of capillaries for different flow categories.

Asphyxiated newborn infants $(n=28)$; during hypothermia day $1=$ white bar; after rewarming day $4=$ gray bar. Healthy normothermic controls $(n=8)=$ black bar.

four velocities and a corresponding increase in category five velocities (brisk flow). There was no difference in mean capillary flow-categorical velocity (MFCV) between the two groups (controls: 3.49 (0.37) vs. patients d4: $3.64(0.68)$ ). Cooled patients on the other hand had marked changes in velocities (Figure 3). MFCV was significantly reduced during cooling compared with after rewarming (d1: $3.34(0.67)$ vs. $\mathrm{d} 4: 3.64$ (0.68), $P<0.05)$.

DRS. Microvascular oxygen extraction was significantly increased on $\mathrm{d} 1$ as compared with $\mathrm{d} 3$, and on $\mathrm{d} 1$ compared with $\mathrm{d} 4$ (Figure 4). There was no difference between the asphyxiated infants on $\mathrm{d} 3$ or $\mathrm{d} 4$ and the healthy controls. No differences in the heterogeneity of the repeated DRS measurements (microvascular oxygen saturation) from twelve nearby measuring volumes, expressed as $\mathrm{CoV}$, was found (patients $\mathrm{d} 1$ : $10.7 \%(4.9-30.0), \mathrm{d} 3: 10.7 \%$ (4.1-23.7), d4: 7.3\% (1.9-26.3), and controls: $12.0 \%(2.2-26.0))$.

\section{DISCUSSION}

Previously, we have demonstrated that the combination of LDPM, CAVM, and DRS may be used to achieve reproducible microvascular data from the skin of healthy newborns (8). The present study showed significant increases in skin functional capillary density and oxygen extraction, as well as reductions in capillary flow velocities and laser Doppler perfusion, during $\mathrm{TH}$.

During cooling, the infant's mean core temperature was reduced to $33.5{ }^{\circ} \mathrm{C}$, causing a reduction in metabolic rate of approximately $20-30 \%$ (6). The approximately $30 \%$ reduction found in LDPM perfusion during cooling is in accordance with the expected reduction in the metabolic demand (Figure 1). It is well recognized that skin LDPM in adults does not reflect skin nutritional perfusion (papillary capillary perfusion), because the larger part of the signal originates from deep skin thermoregulatory plexus perfusion (9). In newborns, the vascular architecture is not fully differentiated, and the epithelium is thinner. The LDPM values may therefore better reflect the metabolic rate of the skin.

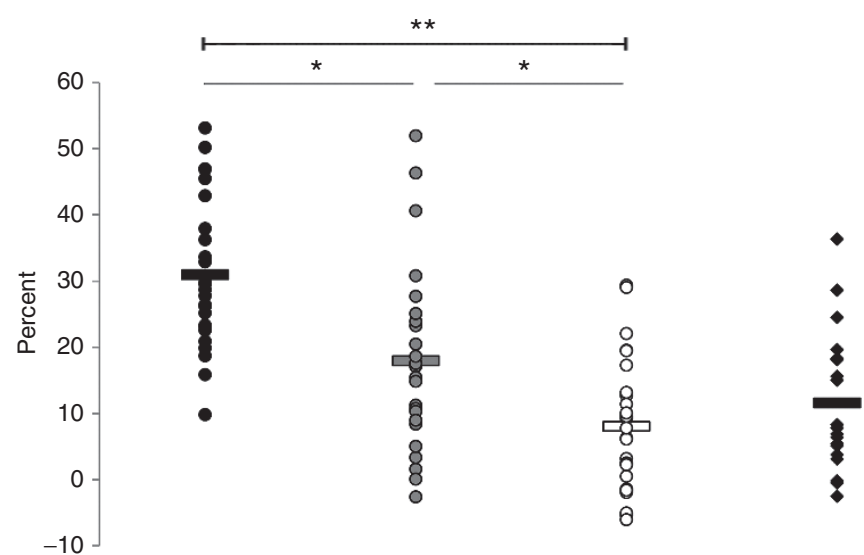

Figure 4. Microvascular oxygen extraction. Asphyxiated newborn infants $(n=28)$; during hypothermia day $1=$ black circle; during hypothermia day 3 = gray circle; after rewarming day $4=$ white circle. Healthy normothermic controls $(n=20)=$ black route. ${ }^{* *} P<0.001,{ }^{*} P<0.01$.

In the year 1919, August Krogh (Nobel prize in Medicine, 1920) postulated that all cells must be located within a critical distance (Krogh diameter) of a perfused capillary to receive a sufficient oxygen supply (10). The oxygen diffusion capacity is reduced with lower temperatures (11). This change in diffusion capacity leads to a reduction in the Krogh diameter, and explains the observed increase in FCD (Figure 2). The high FCD during TH was maintained following rewarming. The reason can be that the initial hypoxic-ischemic event has disturbed the physiological mechanisms regulating microvascular flow distribution. Another possibility is that one third of the patients were developing sepsis, known to affect peripheral blood flow distribution (12).

Delivery of oxygen from a single capillary is dependent on capillary flow velocity and oxygen extraction. The healthy reference population had $62 \%$ of capillaries with flow category four and $36 \%$ in category three, similar to other healthy groups examined by our laboratory $(13,14)$. This velocity distribution in healthy individuals represents the optimal velocities for oxygen delivery. Low velocities give a long erythrocyte capillary passage time, resulting in a high extraction. In spite of high extraction, the oxygen delivery will suffer because of the limited number of erythrocytes passing through the capillary. On the other hand, in a capillary with high flow velocity the number of passing erythrocytes is high, but the passage time may be too short for loading off oxygen. The capillary may be transformed to a physiological shunt with suboptimal oxygen delivery (15).

During $\mathrm{TH}$, asphyxiated patients had marked changes in the capillary flow velocity distribution. Twenty-five percent of the capillaries were in category 1,2 , or 5 with limited capacity for oxygen delivery. We believe that the velocity changes found in this study reflect the reduced metabolism during TH. Rewarmed patients had on $\mathrm{d} 412 \%$ of the capillaries in flow category 5 , a flow category never seen in reference populations of healthy individuals $(8,13,14)$. This finding may be explained by disturbed microvascular regulation secondary to the initial hypoxic-ischemic event or that one third of the patients was developing sepsis during $\mathrm{TH}$ (12). 
On $\mathrm{d} 1$ asphyxiated patients had increased lactate levels caused by the initial hypoxic-ischemic event, and on $\mathrm{d} 3$ lactate was normalized (Table 2). The microvascular data shows the mechanism behind the pay back of this oxygen debt; the increased erythrocyte capillary passage time (reduced MFCV) on d1 gives time for increased oxygen extraction, and the DRS data shows increased microvascular oxygen extraction on d1 (Figure 4).

In a previous paper where the skin microcirculation in healthy newborn infants also was examined by LDPM, CAVM and DRS, we found increased FCD, increased oxygen extraction and reduced LDPM perfusion at the dorsum of the left hand compared with values at the chest (8). In this previous study, the hand skin temperature was approximately $3^{\circ} \mathrm{C}$ lower than the chest temperature, and represented a model of local hypothermia, while the present study is a model of systemic hypothermia. The lower temperature in both models might have induced a similar reduction in the metabolic rate, and shows that the present set of microvascular parameters are able to describe the microvascular mechanisms resulting in reduced oxygen delivery.

\section{Conclusion}

The present study demonstrated that bedside microcirculatory assessments of the skin can be performed in asphyxiated newborns without interfering with ongoing therapy. The results indicate that a set of microvascular parameters describing functional capillary density, capillary flow velocities, and oxygen extraction may be used to characterize oxygen delivery to the cells in the tissue under investigation. The velocity changes on $\mathrm{d} 1$ and $\mathrm{d} 3$ reflect reduced metabolism and the corresponding decrease in oxygen delivery during cooling.

\section{METHODS \\ Study Population}

This single-center prospective study was performed between January 2010 and December 2011 in a tertiary neonatal intensive care unit at Oslo University Hospital, Ullevål, Norway. During this period, 50 infants fulfilled the Norwegian National Guidelines (16) for TH and received whole-body cooling as standard care in our department. The inclusion criteria for $\mathrm{TH}$ were modified from the TOBY study protocol (17). Criterion A was gestational age $\geq 36 \mathrm{wk}$, with at least one of the following: (i) an Apgar score $\leq 5$ at $10 \mathrm{~min}$; (ii) a need for respiratory support at $10 \mathrm{~min}$ following birth, and (iii) either a $\mathrm{pH} \leq 7.00$ or a base deficit $\geq 16 \mathrm{mmol} / \mathrm{l}$ obtained via either umbilical arterial blood or any blood samples taken within 60 min of birth. Criterion B was moderate to severe HIE, which was determined according to the national guidelines, which is a modification from the classification established by Sarnat \& Sarnat $(16,18)$.

Twenty-eight of the 50 neonates were included in the present study (Tables 1 and 2). Inclusion was consecutive when the investigator (S.F.) was available for performing the measurements.

\section{Reference Population}

During a 6-mo period in 2009, 25 healthy term newborns delivered after uncomplicated pregnancies were enrolled in a study assessing skin microcirculation on days 1,2 , and 3 of life, using the same technologies as in the present study (8). In the absence of a noncooled control group with perinatal asphyxia, the results from day 3 of this study were used as reference data.

\section{Treatment Protocol}

The infants were cooled using a servo-controlled water circulated jacket (CritiCool, MTRE, Yavne, Israel) to a target rectal temperature
Table 3. Flow categories with descriptions

\begin{tabular}{ll}
\hline Flow category & \multicolumn{1}{c}{ Description of flow } \\
\hline 0-No flow & $\begin{array}{l}\text { Erythrocytes visible, no movement } \\
\text { 1-Sluggish flow }\end{array}$ \\
2-Continuous very low flow & $\begin{array}{l}\text { Continuous forward movement, } \\
\text { extremely slow } \\
\text { Continuous forward movement, } \\
\text { 3-Continuous low flow }\end{array}$ \\
4-Continuous high flow & $\begin{array}{l}\text { Continuous forward movement, } \\
\text { mostly rapid } \\
\text { Rapidly moving cells throughout } \\
\text { the entire film sequence }\end{array}$ \\
\hline
\end{tabular}

of $33.5^{\circ} \mathrm{C}$ for $72 \mathrm{~h}$ before being slowly rewarmed to $37.0^{\circ} \mathrm{C}\left(0.5^{\circ} \mathrm{C}\right.$ per hour) (16). Pulse oximetry saturation ( $\mathrm{SaO} 2)$ (Masimo Set, Rad-5v, Irvine, CA) and intra-arterial blood pressure measurement were obtained continuously (HP MI008B, Hewlett-Packard, Palo Alto, CA). Skin temperature was measured using a surface temperature scanner (Omega Medical, Model no. STS-101-C, Stanford, CA) attached to the chest immediately before the measurements.

During TH, all infants received continuous morphine infusion. Sixteen infants (57\%) required inotropic medication (dopamine, dobutamine, and/or epinephrine) to maintain a mean arterial blood pressure of 40-50 mmHg. Twenty-five infants were mechanically ventilated to maintain an oxygen saturation $>90 \%$ (Table 1 ).

\section{Microvascular Examinations}

\section{LDPM.}

Microvascular skin perfusion was assessed using a Moor Blood Flow Monitor (MBF 3D) with Moorsoft (Moor instruments, Axminster, Devon, England). Seven 10-s sequences with no or limited movement artifacts were recorded and analyzed. The output was expressed in a semiquantitative scale of flux (arbitrary unit, AU), defined as the product of the number of moving blood cells and their mean velocity in the measured volume of around $1 \mathrm{~mm}^{3}$.

\section{CAVM.}

A hand-held digital CAVM (Optilia, D1, Sundbyberg, Sweden) with a $250 \times$ magnification lens, resolution $640 \times 480$ pixels and frame rate 15 per second were used to obtain five film-sequences of $10 \mathrm{~s}$. The films were stored on a computer for processing (Macbook Air with software Quick Time Player, version 7.6.6, Apple, Cupertino, CA). A high-quality single frame from each film sequence was used for analysis of the FCD. Because the microvessels in newborns exist as a horizontal disorderly network, the FCD-assessments were defined as the number of microvessels crossing a grid of lines per mm line (c/ $\mathrm{mm}$ ) (19). Xscope software (Iconfactory, Greensboro, NC) was used to create the grids as described previously (8).

Films from 28 infants, in total 168 films, were randomly selected for off-line analysis of the capillary flow velocities. The analyzer was blinded to clinical data. The flow velocity within each capillary was scored using a semiquantitative six-category scale modified from a previously published five-category scale (8) (Table 3 ). The data were expressed as the fraction of capillaries in each flow category. MFCV was subsequently calculated using the following formula: $\mathrm{Fr}$ $(1) \times 1+\operatorname{Fr}(2) \times 2+\operatorname{Fr}(3) \times 3+\operatorname{Fr}(4) \times 4+\operatorname{Fr}(5) \times 5(14)$.

\section{DRS.}

A spectrometer operating within the visible wavelength region (AvaSpec-2048-2, Apeldoorn, The Netherlands) and a tungsten halogen light source (AvaLight-HAL, Apeldoorn, The Netherlands) with an effective spectral range of $450-800 \mathrm{~nm}$ were used for the microvascular oxygen saturation measurements. A custom-built fiber optic probe with a fiber composition of three adjacent illuminating fibers (fiber diameter $400 \mu \mathrm{m}$ ) and one receiving fiber (fiber diameter $400 \mu \mathrm{m}$ ) 
resulting in an emitting-receiving distance for the probe of approximately $800 \mu \mathrm{m}$, were used for the measurements (20). A white polytetrafluoretylene tile (WS-2, Avantes, The Netherlands) enclosed in a black plastic housing was used as reference (8). Twelve spectra were collected from each examination in each neonate on all $3 \mathrm{~d}$. Analyses of the spectra were performed by adapting a tissue model based on a diffusion approximation as implemented by Steven Jacques $(21,22)$. Oxy- and deoxy-hemoglobin were then estimated, and microvascular oxygen extraction was calculated as the difference between the arterial and the microvascular oxygen saturation $\left(\mathrm{SaO}_{2}-\mathrm{SmvO}_{2}\right)$.

\section{Study Protocol}

The microvascular assessments were performed using the skin of the chest, at the midline between the supra-sternal notch and left nipple, within $24 \mathrm{~h}$ following the initiation of TH (d1), the last day of TH (d3) and within $24 \mathrm{~h}$ of rewarming (d4). The examinations were performed in the neonatal intensive care unit in a room with a stable temperature of approximately $21^{\circ} \mathrm{C}$ and dimmed lightning. Each infant was placed in an open incubator and was typically intubated and sedated with morphine. CAVM was recorded first, followed by LDPM and DRS. Baby oil (Natusan) was used as immersion oil for the videomicroscope. All equipment was gently applied on the skin surface.

\section{Statistical Analysis}

The demographic data and the coefficient of variation $(\mathrm{CoV})$ are presented as medians with ranges. All other variables are expressed as the means with standard deviations. For the variables measured at different time points within the same group of patients, the paired $t$-test was conducted to compare means. To compare variables between different groups, the independent samples $t$-test was used. $P$ value $\leq 0.05$ was considered statistically significant. The heterogeneity of the microvascular data was expressed as the $\mathrm{CoV}$ (SD/mean) of repeated measurements. The statistical analyses were performed using SPSS for Windows (Statistical Package for the Social Sciences, version 21.0 SPSS, Chicago, IL).

\section{Ethics}

Written parental consent was provided by each patient's parents. The study was approved by the Regional Committee for Medical and Health Research Ethics, South-Eastern Norway, and the Institutional Review Board of Oslo University Hospital.

\section{ACKNOWLEDGMENTS}

Statistical assistance was provided by Professor Leiv Sandvik, University of Oslo. Lise L. Randeberg (NTNU, Trondheim) provided technological support.

\section{STATEMENT OF FINANCIAL SUPPORT}

The authors did not receive any external financial support.

Disclosure: The authors have nothing to disclose.

\section{REFERENCES}

1. Edwards AD, Brocklehurst P, Gunn AJ, et al. Neurological outcomes at 18 months of age after moderate hypothermia for perinatal hypoxic ischaemic encephalopathy: synthesis and meta-analysis of trial data. BMJ 2010;340:c363.

2. Shankaran S, Pappas A, McDonald SA, et al.; Eunice Kennedy Shriver NICHD Neonatal Research Network. Childhood outcomes after hypothermia for neonatal encephalopathy. N Engl J Med 2012;366:2085-92.
3. Jacobs SE, Berg M, Hunt R, Tarnow-Mordi WO, Inder TE, Davis PG. Cooling for newborns with hypoxic ischaemic encephalopathy. Cochrane Database Syst Rev 2013;1:CD003311.

4. Kasdorf E, Perlman JM. Strategies to prevent reperfusion injury to the brain following intrapartum hypoxia-ischemia. Semin Fetal Neonatal Med 2013;18:379-84.

5. Al Zaabi A, Rahmani AY, Souid A. Optimal temperature for whole-body hypothermia in the newborn: an in vitro study using foreskin mitochondrial oxygen consumption. J Neonatal Perinatal Med 2014;7:179-83.

6. Laptook AR, Corbett RJ. The effects of temperature on hypoxic-ischemic brain injury. Clin Perinatol 2002;29:623-49, vi.

7. Cecconi M, De Backer D, Antonelli M, et al. Consensus on circulatory shock and hemodynamic monitoring. Task force of the European Society of Intensive Care Medicine. Intensive Care Med 2014;40:1795-815.

8. Fredly S, Fugelseth D, Wester T, Häggblad E, Kvernebo K. Skin microcirculation in healthy term newborn infants-assessment of morphology, perfusion and oxygenation. Clin Hemorheol Microcirc 2015;59:309-22.

9. Mørk C, Kvernebo K, Asker CL, Salerud EG. Reduced skin capillary density during attacks of erythromelalgia implies arteriovenous shunting as pathogenetic mechanism. J Invest Dermatol 2002;119:949-53.

10. Krogh A. The supply of oxygen to the tissues and the regulation of the capillary circulation. J Physiol 1919;52:457-74.

11. Sidell BD. Intracellular oxygen diffusion: the roles of myoglobin and lipid at cold body temperature. J Exp Biol 1998;201(Pt 8):1119-28.

12. De Backer D, Orbegozo Cortes D, Donadello K, Vincent JL. Pathophysiology of microcirculatory dysfunction and the pathogenesis of septic shock. Virulence 2014;5:73-9.

13. Wester T, Awan ZA, Kvernebo TS, Salerud G, Kvernebo K. Skin microvascular morphology and hemodynamics during treatment with veno-arterial extra-corporeal membrane oxygenation. Clin Hemorheol Microcirc 2014;56:119-31.

14. Wester T, Häggblad E, Awan ZA, et al. Assessments of skin and tongue microcirculation reveals major changes in porcine sepsis. Clin Physiol Funct Imaging 2011;31:151-8.

15. Angleys H, Østergaard L, Jespersen SN. The effects of capillary transit time heterogeneity $(\mathrm{CTH})$ on brain oxygenation. J Cereb Blood Flow Metab 2015;35:806-17.

16. Skranes JH, Fugelseth D, Stiris T. Keeping a cool head. Paidos 2011;29: 21-24.

17. Azzopardi D, Brocklehurst P, Edwards D, et al.; TOBY Study Group. The TOBY Study. Whole body hypothermia for the treatment of perinatal asphyxial encephalopathy: a randomised controlled trial. BMC Pediatr 2008;8:17.

18. Sarnat HB, Sarnat MS. Neonatal encephalopathy following fetal distress. A clinical and electroencephalographic study. Arch Neurol 1976;33:696-705.

19. De Backer D, Creteur J, Preiser JC, Dubois MJ, Vincent JL. Microvascular blood flow is altered in patients with sepsis. Am J Respir Crit Care Med 2002;166:98-104.

20. Meglinsky IV, Matcher SJ. Modelling the sampling volume for skin blood oxygenation measurements. Med Biol Eng Comput 2001;39:44-50.

21. Jacques SL. Time-resolved reflectance spectroscopy in turbid tissues. IEEE Trans Biomed Eng 1989;36:1155-61.

22. Farrell TJ, Patterson MS, Wilson B. A diffusion theory model of spatially resolved, steady-state diffuse reflectance for the noninvasive determination of tissue optical properties in vivo. Med Phys 1992;19:879-88. 